\title{
HOW DOES THE MAGNUS FORCE PRODUCED FOR DIFFERENT ANGULAR VELOCITY VARY ACROSS DIFFERENT VALUES OF SURFACE ROUGHNESS SUCH AS FOIL, PLASTIC, PAPER, CLOTH, AND FELT APPLIED ON A ROTATING CYLINDER?
}

\section{DEVANSH RATHI \& DEEPAK KUMAR CHOUDHARY}

Podar International School, Mumbai, Maharashtra, India

\section{ABSTRACT}

When a rotating cylinder was placed inside the uniform flow stream of any fluid, it created a force on the cylinder, called the Magnus force, perpendicular to the flow of the fluid. This force is directly related to the surface roughness of the material of the cylinder and the angular velocity of the cylinder's rotation. In this research paper, I investigated this relation between the Magnus force produced, for different angular velocities and how it varies across the different values of the surface roughness applied to the cylinder

To carry out an experiment, the cylinder was attached to a motor and fixed in such a way that, the cylinder rotated horizontally, and then this setup was stuck with a mass balance. The surface roughness of the cylinder were varied, by wrapping surfaces with different coefficient of static friction onto the cylinder, and the angular velocity was varied by varying the voltage across the motor. Using a hair-dryer, fixed at a constant distance away from the spinning cylinder, a uniform flow stream was provided. This resulted in a Magnus force that acted in the upward direction on the cylinder, which was calculated by measuring the change in mass of the setup, that was stuck to the mass balance.

The angular velocity and the Magnus force had a linear relationship. This was due to the fact that, a higher value of angular velocity causes a bigger vortex, which results in a thicker boundary layer around the cylinder. This results in a stronger Magnus force. The force was directly proportional to the root of the coefficient of static friction of the material. Since, higher values result in stronger friction forces between the surface of the cylinder and the air particles, increasing the width of the boundary layer.

KEYWORDS: Cylinder, Boundary Layer \& Magnus Force

Received: Jul 22, 2017; Accepted: Aug 13, 2017; Published: Aug 30, 2017; Paper Id.: IJPROCT20171

\section{INTRODUCTION}

Magnus force is a force that is experienced by spinning cylindrical or spherical solids, while it's submerged inside a fluid, which might be a gas or a liquid. This force, named after Gustav Magnus is the man who first discovered this effect, while investigating how cannon balls propagated through the air.

The effect of the Magnus force can be explained, as the ball in the air, spinning clockwise creates a downward Magnus force, and if ball spinning anti-clockwise acts on by an upward force, this extends or shortens the horizontal distance travelled respectively, while the non-spinning ball is unaffected. 


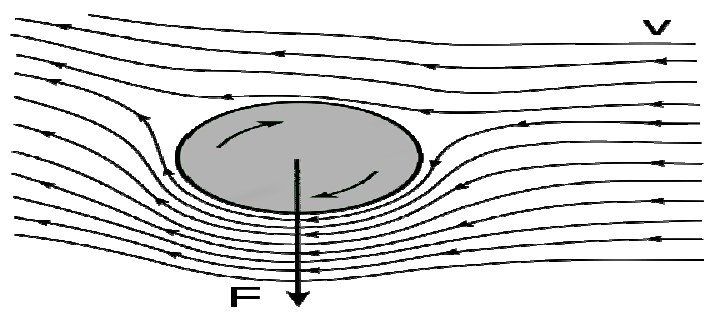

Figure 1

The Magnus force can be explained by the superposition of the vortex, created by the rotating cylinder and the uniform air flow as can be seen in Figure 1. A simplified explanation of the force would be that, due to the friction between the surface of the cylinder, the air is 'dragged' along, on the side of the cylinder and tangential velocity is moving with that of the uniform air flow. In the case of Figure 1, this is the bottom side. This results in an increase in air speed on the bottom side of the cylinder. Near the top side, however, the air slows down due to friction opposing the airflow.

The Bernoulli's principle states that, increasing the flow velocity of any fluid lowers the fluid pressure in that region. This implies that, due to the higher airspeed in the bottom part of the cylinder, the pressure decreases on the bottom part and increases on the top, due to slower speeds. This pressure difference is what pushes the cylinder downwards. This force is always perpendicular to the velocity vector of the air and the angular velocity vector (Figure 1). The angular velocity vector is always normal to the cross-section of the cylinder

This does not include the viscous nature of the fluid and the cylinder rotating in. Due to this and friction, a 'noslip' condition is formed, which results in a boundary layer being formed in which air near the boundary rotates with the cylinder. As Swanson explained, the spinning causes an asymmetric flow in the air and causes the boundary layer to be separate at different points, as can be seen in Figure 1. This is what created the vortex needed for the Magnus force. Since, the cylinder is producing an upward force on the air particles, according to the Newton's third law, as studied in our class, there will be an equal and opposite reaction, which would give rise to the downward force on the ball.

This force is mathematically denoted by the Kutta-Joukowski theorem ${ }^{1}$ for a cylinder:

$F_{m}=\rho G v L$ Where,$F_{m}=$ Magnusforce, $\rho=$ Densityofthemedium, $G=$ Vortexstrength

$v=$ Velocityof themedium, $L=$ Lengthofthecylinder

In this equation Vortex strength, $\mathrm{G}$, is given by:

$G=2 \pi \omega r^{2}$ Where, $\omega=$ Angularvelocityinrad $/ \mathrm{sec}, r=$ radiusofthecylinder

The vortex strength does depend on the surface roughness of the cylinder, and the above equation is a simplified version. Another, more complicated, equation found also takes into account, the drag experienced ${ }^{2}$ :

$\overrightarrow{F_{m}}=\frac{1}{2} C_{L} \rho A v^{2}(\vec{\omega} \times \vec{v})$

Where, $C_{L}=$ Liftcoefficient,${ }^{\prime} C_{L}{ }^{\prime}$ is the value that changes according to surface roughness

\footnotetext{
1"Lift of A Rotating Cylinder". Grc.nasa.gov. N.p., 2017. Web. 4 Feb. 2017.

${ }^{2}$ Richmond, Michael. "The Effect Of Air On Baseball Pitches". Spiff.rit.edu. N.p., 2008. Web. 4 Feb. 2017.
} 


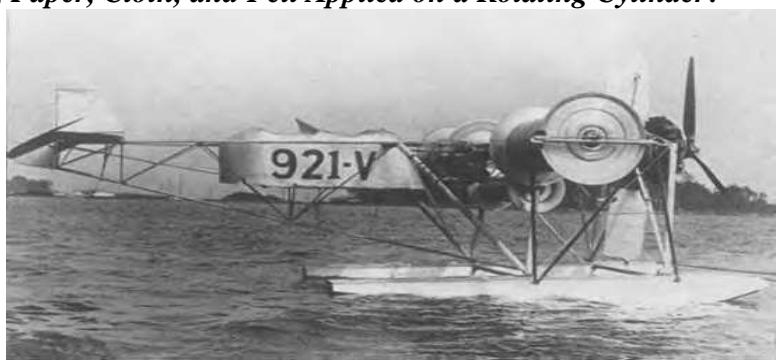

Image 1

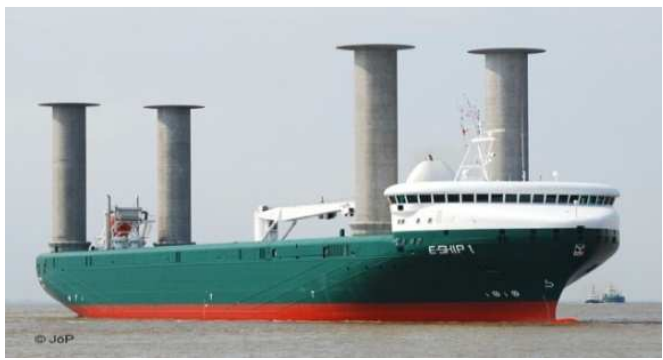

Image 2

This phenomenon is essential for ball sports, like football and cricket, where a spin to the ball is purposely introduced to make the ball 'curve' to the desired target. There are practical ships that use this phenomenon to propel themselves forward called Flettner Rotor ships (Image 1$)^{3}$. There are also wings that use these rotating cylinders for providing the lift force necessary, and could one day be used for practical air crafts (Image 2$)^{4}$.

Higher surface roughness leads to less drag force, since it delays the separation of the boundary layer inducing turbulent (chaotic) flow, which is less prone to this separation that, laminar (smooth) flow is more stable. This dramatically increases the drag. To achieve this greater roughness, golf balls are seen to have 'dimples' on its surface. This decreased drag would give these spinning wings, an edge over the traditional wings.

\section{Initial Experimental Design}

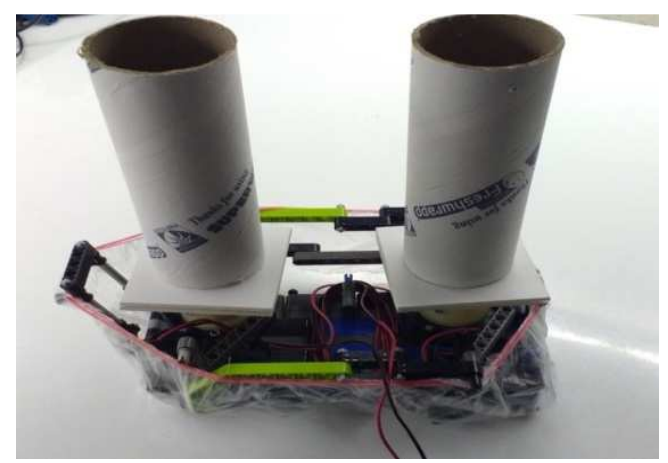

Image 3

My initial experimental design was to recreate the Flettner rotor ship (Image 3). The plan was to measure the time the shipped takes to travel 1 meter and then using the kinematics formula, taught in the IB curriculum, to calculate the acceleration. Thus, by using $F=m a$, the Force

\section{This Design was rejected Due to the Following Problems}

- The biggest source of error was the fact that, at higher rotational speeds the vessel started wobbling due to the moment of inertia of the cylinders. This resulted in, the boat not travelling in a straight trajectory, thus the data collection proved to be difficult

- Even though identical motors were used, the rotation speeds differed to up to $20 \%$. This could have led to uneven force from the cylinders, and a torque being created

\footnotetext{
3"Anton Flettner Rotor Vessel Ship Backau, Now Renamed Baden-Baden". Thiiink.com. N.p., 2017. Web. 4 Feb. 2017.

4"The Spinning Wing".Pilotfriend.com. N.p., 2017. Web. 4 Feb. 2017.
} 


\section{Modified Experimental Design}

After further revision of the problems faced with initial design, a better method of investigation was designed to tackle the research question. To avoid too many moving parts, I decided to keep as many parts as possible fixed in their place. Also, instead of the two motors, with uneven angular velocities, I designed this experiment only to require one.

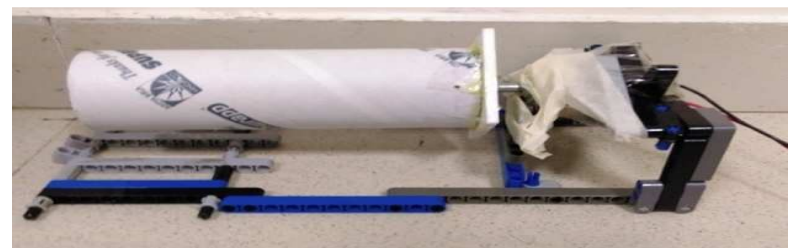

Image 4

Instead of emulating the ship, I decided to emulate the spinning wing. To do this, I used Mechanical Legos® to create a stand, to place the cylinder such that it rotates horizontally (Image 4). A silicon based adhesive material was used to fasten everything in place, such that nothing would start loosening after some time of usage. To further avoid the wobble experienced last time, I fixed the motor to the base at both the ends.

The independent variable is the different angular velocities of the motor, and the dependent variable is the upward lift force exerted by the rotation of the cylinder, which would be repeated for different materials. To measure this force, the entire setup showed in Image 4, was taped down to a mass balance, as can be seen in Image 5 (below). The mass balance was tarred to the mass of the machine, such that the mass balance showed a reading of ' 0.00 ' when the machine was taped on it.

The motor was connected to the power supply such that, the cylinder was rotating clockwise. Thus, when the air supply and the power supply were switched on, the Magnus effect took place, creating a lift force which results in the mass reading, to go into the negatives. This mass readings can then be used, to calculate the force exerted by the spinning cylinder.

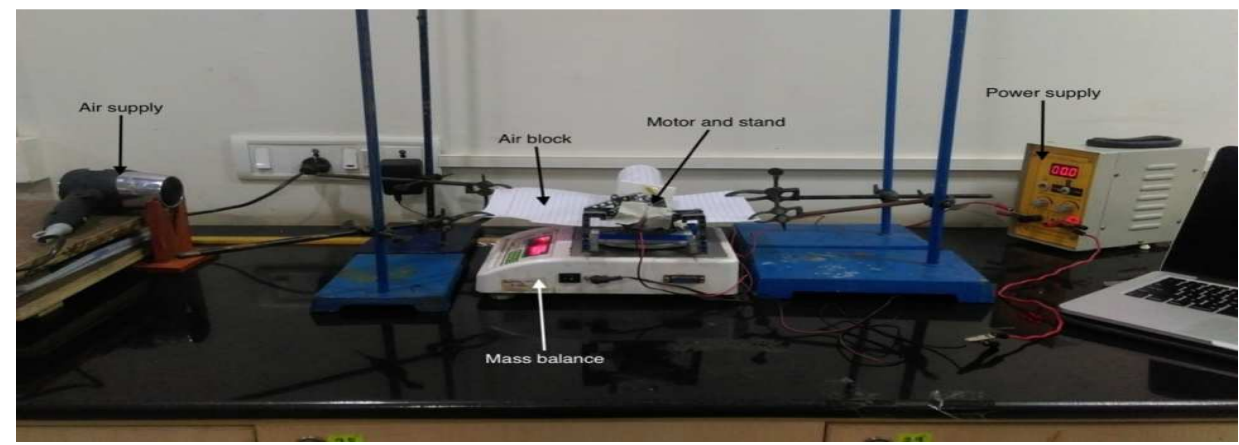

Image 5

\section{Apparatus, Used}

- $\quad$ Mass balance $( \pm 0.01 \mathrm{~g})$, Power supply $( \pm 0.1 \mathrm{~V})$, Motor (rated 15V), Hair dryer, Clamps, Stands, V-block

- $\quad$ Paper cylinder (radius of $2.66 \mathrm{~cm}$ ), Pieces of: Foil, Plastic, Paper, Cloth, Felt (rough textile)

\section{Procedure}

- Set up the circuit and apparatus as shown in Figure 4 and Image 5. Wrap the cylinder with the material to be 
tested. The stand and the motor should be placed on the mass balance and then the balance should be tarred such that, a reading of ' 0.00 ' is shown. The air supply should be placed $50 \mathrm{~cm}$ away from the center of the cylinder, and should be placed in such a way that, it's perfectly at the same height as the cylinder is placed and the wind is hitting the cylinder (the angle of attack) at perfectly 90 degrees. Switch on the power supply and set the voltage of the power supply to $1.5 \mathrm{~V}$. Switch on the air supply. Let this go on for around 10 seconds, such that the value being seen on the mass balance stabilizes. This is needed, since it takes time for the stream of air around the cylinder to start moving and stabilize at a constant speed. After 10 seconds, start taking a video recording of the readings of mass balance, for the next 7 seconds. This is done since the readings tend to fluctuate a bit. Then analyze the video, by taking the maximum and the minimum mass readings achieved in these fluctuations. Then switch off the power and air supply. If the readings do not go back to '0.00', then tare it again after it falls down and stabilizes. Then repeat the entire process 4 times. Repeat this process for $3-15 \mathrm{~V}$, with a $1.5 \mathrm{~V}$ increment every time. Again, repeat this entire process for all the selected materials.

\section{Variables}

\section{Independent Variable: Angular Velocity}

The angular velocity of the cylinder was changed so that, a wider range of data can be used to compare the data between every surface. Since, the motors were rated ' $15 \mathrm{~V}$ ', I took 11 different voltages ranging from 0 to 15 Volts. The angular velocities achieved at every voltage were then calculated, by sticking a small piece of paper such that it was projecting outwards from the cylinder at about $1 \mathrm{~cm}$. After switching on the motor and setting it at the desired voltage, a firmly fixed piece of metal was introduced such that, it just grazed the paper that is projecting outwards, creating a sound everytime the paper reached that particular point while rotating. The noise was then recorded and analyzed using Audacity ((sound-analyzing software). An example can be seen below (Image 6):

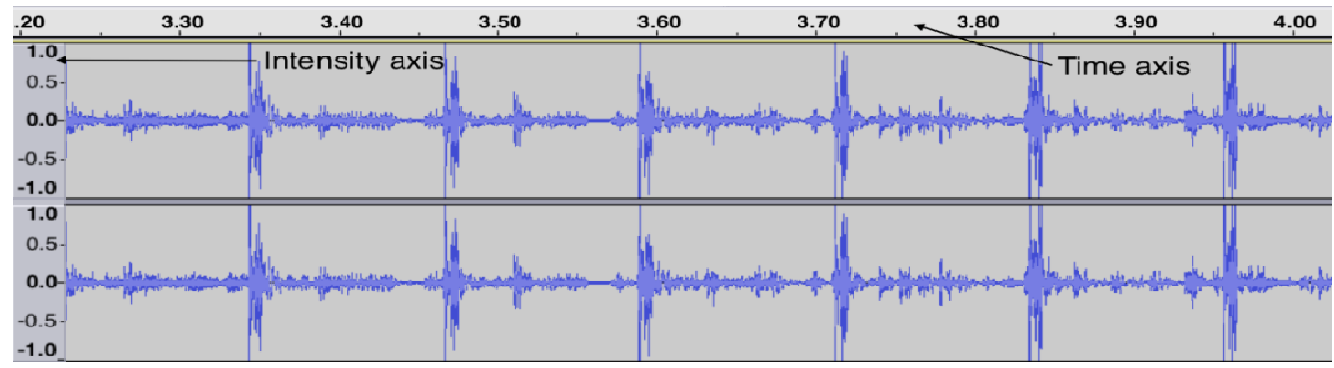

Image 6

The peaks in intensity occur when the paper projectile hits the piece of the metal. Using the software and measuring the time between each peak, the time period $T$, for one rotation was found. To find the angular velocity, $\omega$ the following formula, taught in the IB curriculum, was used: $\omega=\frac{2 \pi}{T}$

The readings from the software were in 3 decimal places. The following data were obtained

Table 1

\begin{tabular}{|c|c|c|c|c|c|}
\hline \multirow{2}{*}{ Voltage/V } & \multirow{2}{*}{ Starting Time/ s } & \multirow{2}{*}{ Ending Time/ s } & \multirow{2}{*}{ Time Period/ s } & \multicolumn{2}{|c|}{ Rotational Frequency } \\
\cline { 4 - 6 } & & & & 0.000 & 0.000 \\
\hline 0 & & & 0.646 & 9.726 & 1.548 \\
\hline 1.5 & 5.769 & 6.415 & 0.269 & 23.358 & 3.717 \\
\hline 3 & 3.404 & 3.673 & $\mathbf{R}^{-1}$ & RPS/ rev s \\
\hline
\end{tabular}




\begin{tabular}{|c|c|c|c|c|c|}
\hline 4.5 & 3.251 & 3.417 & 0.166 & 37.851 & 6.024 \\
\hline 6 & 6.531 & 6.653 & 0.122 & 51.502 & 8.197 \\
\hline 7.5 & 5.265 & 5.361 & 0.096 & 65.450 & 10.417 \\
\hline 9 & 6.997 & 7.073 & 0.076 & 82.673 & 13.158 \\
\hline 10.5 & 6.285 & 6.35 & 0.065 & 96.664 & 15.385 \\
\hline 12 & 6.239 & 6.297 & 0.058 & 108.331 & 17.241 \\
\hline 13.5 & 2.647 & 2.698 & 0.051 & 123.200 & 19.608 \\
\hline 15 & 3.59 & 3.636 & 0.046 & 136.591 & 21.739 \\
\hline
\end{tabular}

\section{Controlled Variables}

\section{Surface Roughness}

The surface used was changed across a different set of experiments so that, variation with respect to the surface could be observed.

To actually measure the surface roughness of any material, it requires expensive apparatus that was outside the limits of this essay. Instead, coefficient of static friction $\left(\mu_{\mathrm{s}}\right)$ of different materials was used. This was done since $\mu_{\mathrm{s}}$ can be found using a simple setup (Image 5). Static friction was used (and not dynamic friction) because, the boundary layer that is formed moves with the surface of the cylinder, which means Magnus force works because there is no relative motion between the air particles and the surface.

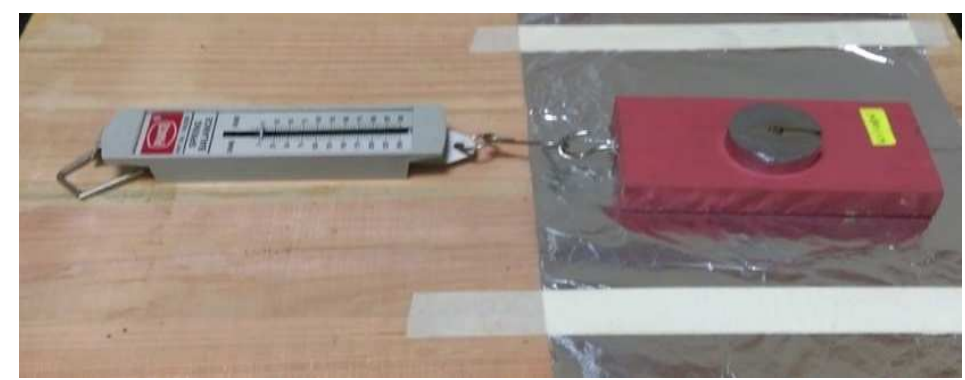

Image 7

The mass balance was slowly pulled away from the block, till there was enough force to overcome the static friction of the block. The surface whose coefficient of static friction is to be found was taped down as seen above. A slowmotion video was taken, to accurately see at what reading does the block starts to move. This is a modified version of a lab, that was conducted during the course of IB. And, as per that lab, the following formula was used to find the coefficient.The same block of mass 199.4g was used for every trial: $f=\mu_{s} N, \mu_{s}=\frac{f}{\left(\frac{199.4}{1000} \times 9.81\right)}$

A variety of surfaces were tested to get a good range of the coefficient of static frictions. The chosen ones are seen below: (all the readings of all trials are shown in the appendix table 1)

Table 2

\begin{tabular}{|l|c|}
\hline Material & Average $\mu_{\text {s }}$ \\
\hline Foil & 0.27 \\
\hline Plastic & 0.42 \\
\hline Paper & 0.56 \\
\hline Cloth & 0.70 \\
\hline Felt & 0.81 \\
\hline
\end{tabular}


Since the Magnus force always acts perpendicular to the direction of the flow of the air stream, the angle of attack should be fixed at $90^{\circ}$. This matched the height of the contours of the air supply and cylinder, using wooden boards and the v-block.

\section{Wind Speed}

This was done by placing the air supply $\mathbf{5 0} \mathbf{~ c m}$ away, at the same settings for every trial. The wind speed was not calculated, since I did not have access to an anemometer, the hair dryer was kept at the same settings throughout the experiment.

\section{Background Disturbances}

Even smaller disturbances can result in a significant difference in the data. To minimize the disturbances, the windows and doors were closed, the experiment was placed away from any source of a draft, and all unnecessary movements were avoided.

\section{Reading Without any Spin}

Even when the cylinder is not spinning (the $0 \mathrm{~V}$ reading), switching on the air supply creates a force on the entire setup of the rotating cylinder, and due to the setup not being perfectly symmetrical, it causes a force not perfectly horizontal. This results in this force altering the recorded mass of the setup. To avoid this, before starting the data collection, 4 trails were taken with no rotation for every material, and the reading was then subtracted from the rest of the results, since this reading would have existed independent of the Magnus force.

\section{Temperature}

The density of the air has a directly proportional relationship to the Magnus force, so it had been held constant throughout the experiment. To do so, the room was air conditioned at a constant value of $24^{\circ} \mathrm{C}$, which resulted in an air density of $1.189 \mathrm{~kg} / \mathrm{m}^{3}$.

\section{Same Apparatus}

The apparatus used to be the same always. This was done to ensure that, even if there are any inaccuracies in the instruments used, the inaccuracies remain constant for all the trails so that, they can be compared. Eg. The power supply, air supply, mass balance, etc.

\section{Forces on the Weighing Scale}

Since the air is pushed downward in this experiment, it could result in the air being pushed down and exerting a force of the scale, resulting in a lower estimation than the real value. To tackle this, a sheet of paper was suspended in such a way that it does not make contact with the setup but acts as a block against the pushed air. This can be seen in Image 5 .

\section{Primary Data Collection}

A total of 240 readings was taken, divided amongst 6 different tested materials, as shown in tables 3, the example of the foil, similarly readings of other surface has been taken. 
Table 3

\begin{tabular}{|c|c|c|c|c|c|c|c|c|}
\hline \multicolumn{10}{|c|}{ Foil } \\
\hline \multirow{3}{*}{ Voltage/V } & \multicolumn{9}{|c|}{ Mass Reading/g } \\
\cline { 2 - 10 } & \multicolumn{2}{|c|}{ Trial 1 } & \multicolumn{2}{c|}{ Trial 2 } & \multicolumn{2}{c|}{ Trial 3 } & \multicolumn{2}{c|}{ Trial 4 } \\
\cline { 2 - 9 } & Max & Min & Max & Min & Max & Min & Max & Min \\
\hline 1.5 & 1.17 & 0.68 & 1.24 & 0.71 & 1.17 & 0.68 & 1.19 & 0.68 \\
\hline 3.0 & 2.02 & 1.55 & 2.06 & 1.50 & 2.00 & 1.51 & 2.01 & 1.52 \\
\hline 4.5 & 2.40 & 1.83 & 2.47 & 1.84 & 2.38 & 1.92 & 2.41 & 1.93 \\
\hline 6.0 & 2.66 & 2.16 & 2.70 & 2.18 & 2.72 & 2.19 & 2.64 & 2.22 \\
\hline 7.5 & 3.28 & 2.79 & 3.24 & 2.77 & 3.32 & 2.82 & 3.27 & 2.78 \\
\hline 9.0 & 3.91 & 3.43 & 3.94 & 3.46 & 3.98 & 3.41 & 3.96 & 3.41 \\
\hline 10.5 & 4.30 & 3.77 & 4.38 & 3.81 & 4.29 & 3.72 & 4.27 & 3.71 \\
\hline 12.0 & 4.90 & 4.42 & 4.95 & 4.33 & 4.90 & 4.35 & 4.84 & 4.31 \\
\hline 13.5 & 5.34 & 4.78 & 5.40 & 4.87 & 5.30 & 4.79 & 5.39 & 4.73 \\
\hline 15.0 & 5.90 & 5.35 & 5.98 & 5.33 & 6.00 & 5.40 & 5.79 & 5.25 \\
\hline
\end{tabular}

\section{Data Analysis}

Due to the amount of data points, not all the data processing will be shown. However, the same calculations seen for the material Foil were done for all other materials too.

Table 4: 0V Reading: 0.62g

\begin{tabular}{|c|c|c|c|c|c|c|c|}
\hline \multicolumn{9}{|c|}{$\begin{array}{c}\text { Angular } \\
\text { Velocity/Rad s- } \\
\text { 1 }\end{array}$} & $\begin{array}{c}\text { Average } \\
\text { Readings/ g } \\
\text { Max }\end{array}$ & \multicolumn{1}{c|}{$\begin{array}{c}\text { Mass } \\
\text { Reading/g }\end{array}$} & $\begin{array}{c}\text { Uncertainty } \\
\text { in Mass }\end{array}$ & $\begin{array}{c}\text { Mass } \\
\text { Accounting for } \\
\text { 0V Readings }\end{array}$ & Force/N & $\begin{array}{c}\text { Uncertainty } \\
\text { in Force }\end{array}$ \\
\hline 9.73 & 1.19 & 0.68 & 0.94 & 0.25 & 0.32 & 0.00309 & 0.00248 \\
\hline 23.36 & 2.02 & 1.52 & 1.77 & 0.25 & 1.15 & 0.01124 & 0.00246 \\
\hline 37.85 & 2.41 & 1.88 & 2.14 & 0.27 & 1.52 & 0.01494 & 0.00262 \\
\hline 51.50 & 2.68 & 2.18 & 2.43 & 0.25 & 1.81 & 0.01774 & 0.00242 \\
\hline 65.45 & 3.27 & 2.79 & 3.03 & 0.24 & 2.41 & 0.02363 & 0.00239 \\
\hline 82.67 & 3.94 & 3.42 & 3.68 & 0.26 & 3.06 & 0.03004 & 0.00255 \\
\hline 96.66 & 4.31 & 3.75 & 4.03 & 0.28 & 3.41 & 0.03342 & 0.00273 \\
\hline 108.33 & 4.89 & 4.35 & 4.62 & 0.27 & 4.00 & 0.03924 & 0.00267 \\
\hline 123.20 & 5.35 & 4.79 & 5.07 & 0.28 & 4.45 & 0.04365 & 0.00277 \\
\hline 136.59 & 5.91 & 5.33 & 5.62 & 0.29 & 5.00 & 0.04905 & 0.00287 \\
\hline
\end{tabular}

The voltage readings were replaced by the respective angular velocity as found above (Table 1). The column with the title 'Mass accounting for $0 \mathrm{~V}$ readings' is found by simply subtracting the $0 \mathrm{~V}$, found before the trials of all the materials. The adjusted readings were the ones that were carried on, to find the force exerted.

Using the formula learnt in the IB curriculum, $W=m g$, and rearranging it. The following formula was obtained, to calculate the Magnus force exerted by the cylinder. The mass is divided by 1000 , since the readings were obtained in grams. $F_{M}=\frac{\Delta m}{1000} \times 9.81$

The mass reading was calculated by taking the mean value of the maximum and minimum reading. And, the uncertainty was calculated by the following formula: Uncertainty $=\frac{\operatorname{MaxF}_{m}-\operatorname{MinF}_{m}}{2}$

All of the data and the uncertainties were inputted into a spreadsheet software and then plotted onto a graph, and suitable trend line was plotted, for all different materials. The following graph was obtained: 


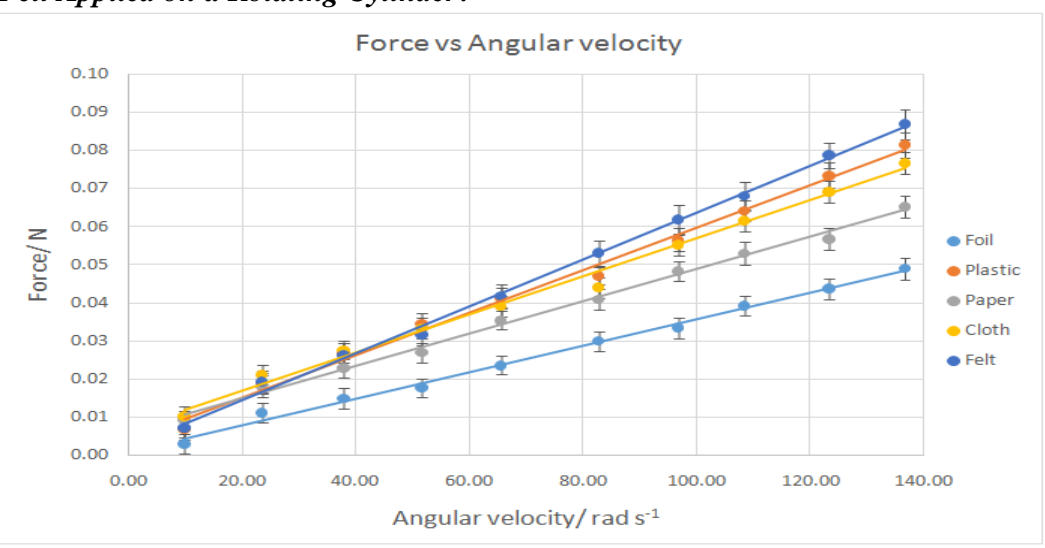

Figure 1

As can be clearly seen from Figure 4, angular velocity and force exerted have a linear relationship. This fits with the predictions made by the Kutta-Joukowski theorem, as shown above. However, not all the data fit a linear trend, as not all of the trend lines extends to meet the origin, since the equations of the lines are seen to have some non-zero y-intercept. There is no observable trend in how much the trend lines, stray away from the origin, so I concluded that, this was nothing more than random errors in experimentation.

This graph also confirms that, $\mu_{\mathrm{s}}$ and the Magnus force have a directly proportional relationship, to some extent. This is due to the fact that, the material plastic acts like an outlier and does not fit in with the trend. Also, at the lower values of angular velocity, everything is random and does not fit in with any trend. This could be due to the same random errors. The uncertainty values are lower than I had initially expected it to be, after the initial experiment design. So, fixing the motor from both the ends was quite effective in decreasing the wobble experienced. Though it is hard to see the difference from the graph, a closer look at the data tables will show that, at higher angular velocities the uncertainty tends to be higher than lower ones. This could be due to the fact that, at higher angular velocities the wobble increases and due to air particles moving faster near the surface of the cylinder, the flow would be chaotic and might have caused divisions.

To get a better look at the relationship between $\mu_{\mathrm{s}}$ and the Magnus force, the readings at the highest angular velocity $\left(\mathbf{1 3 6 . 5 9 1} \mathbf{r a d ~ s}^{-1}\right)$ and plotted against $\mu_{\mathrm{s}}$, in a different graph and table is shown below

Table 5

\begin{tabular}{|l|c|c|c|}
\hline Material & $\boldsymbol{\mu}_{\mathbf{s}}$ & Force/ N & Uncertainty in Force \\
\hline Foil & 0.27 & 0.04905 & 0.00287 \\
\hline Plastic & 0.42 & 0.08141 & 0.00325 \\
\hline Paper & 0.56 & 0.06521 & 0.00280 \\
\hline Cloth & 0.70 & 0.07670 & 0.00298 \\
\hline Felt & 0.81 & 0.08683 & 0.00391 \\
\hline
\end{tabular}




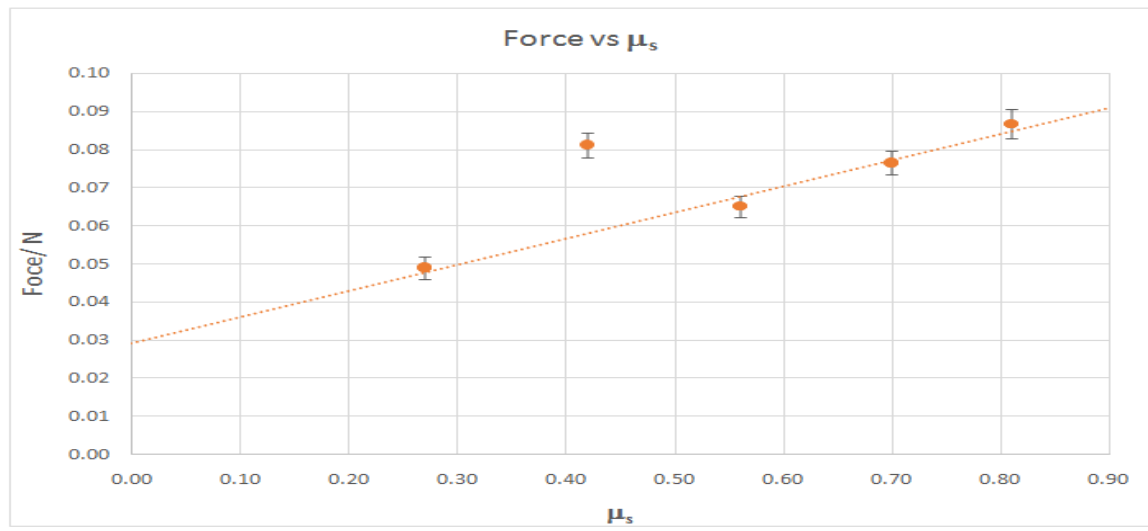

Figure 2

As can be seen from Figure 5, plastic acts like an outlier and thus, has been excluded in the sketching of the trend line. This unusual increase in the force for the recorded $\mu_{\mathrm{s}}$ could have been because of the creases that were formed, when it was wrapped around the cylinder, which were not present during the measurement of the $\mu_{\mathrm{s}}$. These creases could have caused the boundary layer formed to be thicker, resulting in a stronger force.

The uncertainty is higher at higher levels of $\mu_{\mathrm{s}}$. This difference, again, cannot be seen in the graph above, but a closer analysis of the tables reveals that, there is a slight increase in rougher materials like the felt and the cloth.

To get a better understanding of the relationship, the following graph was plotted:

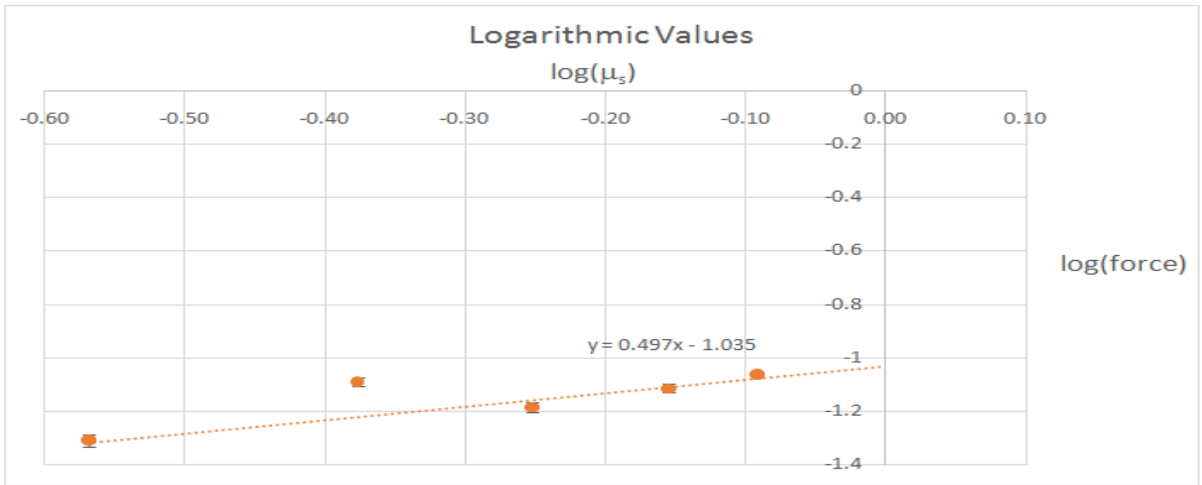

Figure 3

The uncertainties for this graph were calculated, by taking the max and min readings of every data point and calculating the force from them. Then, the following formula was used: Uncertainty $=\frac{\log (\max \text { Force })-\log (\min \text { Force })}{2}$

But, the uncertainties are almost negligible as can be seen in Figure 6, so are simply ignored.

Again, for the data point for plastic is excluded during the calculation of the best fit line. Using the spreadsheet software, the equation of the line was generated (seen on the graph), which is: $y=0.4979 x-1.0351$

\section{CONCLUSIONS}

Taking logarithms results in the following mathematics: $F_{m} \propto \mu_{s}{ }^{n}$

$F_{m}=k \mu_{s}{ }^{n}$ further processed using LOG to linearize, the equation becomeslog $\left(F_{m}\right)=n \log \left(\mu_{s}\right)+\log (k)$ 
This can now be compared to equation of the line obtained from Figure 6, to get a value of ' $n$ ', as the gradient would now be ' $n$ ' since the y-axis corresponds to ' $\log \left(F_{m}\right)$ ' and $\mathrm{x}$-axis corresponds to ' $\log \left(\mu_{s}\right)$ '.

Therefore, we get the value of ' $n$ ' to be 0.4979 , which, accounting for error could be rounded off to 0.5 . This would imply that, there is a square root relationship between the coefficient of static friction (which is a way I have used to approximate the surface roughness) and the Magnus force, which is: $F_{m} \propto \sqrt{\mu_{s}}$

This relationship was surprising, as it seemed like it would be a linear relationship judging from Figure 5. This could have not been apparent without the logarithmic graph as a range of values was not large enough to make the shape of the graph apparent. The directly proportional relation was expected, as a higher surface roughness (higher $\mu_{s}$ ) Would mean that it would take more force to separate the air particles from the surface of the cylinder. Since this friction force is what acts as the centripetal force for the boundary layer, a higher $\mu_{s}$ Would mean thicker boundary layer, which would result in a higher Magnus force.

The relationship of the angular velocity and the Magnus force is a linear relationship, which was expected since a higher revolution rate would result in a larger vortex strength, which would result in more air being pushed downwards. The linear relationship is predicted by the Kutta-Joukowski theorem, but this is not perfect as it does not give the relation between the surface roughness and the Magnus force.

The anomalies in the material foil due to the creases formed raised a question about the effect of different shapes used for the rotor. Since there was such a large increase in the Magnus force due to the irregularities. These irregularities could be artificially made in the form of ridges and could be used to increase the efficiency of such engines. And, an extremely smooth surface could have also been tested to see the effects of the phenomenon known as the reverse Magnus effect (the direction of the force becomes opposite for very smooth surfaces). More about this can be read in (Briggs 594595). Spinning wings such as the one I tested are an efficient way of providing lift and take care of the lot of problems that are faced by traditional wings that are used. The paper (Patkunam, Sigamani and Mahathi) ${ }^{56}$ is a comparative study with the traditional wing and the rotor wing that uses the Magnus force. These spinning wings could replace the traditional wings, since they decrease the experienced drag force, but there are also problems faced with the setup of such wings.

\section{Evaluation}

The experiment mostly fit in with the expected results found online. The data points fit in the trend very well as they only deviate from the trend-line by a small amount. The uncertainties were lesser than they expected, which was the average of $\pm \mathbf{0 . 0 0 2 8 1 N}$ only.

\section{The Major Sources of the Error Were:}

Systematic: The biggest area of improvement would the use of actual measures of surface roughness such as:

- The average of the deviation peaks of troughs from the mean height value, the average of the height difference between the peaks and troughs. These values are given in micrometer or nanometer, usually.

\footnotetext{
${ }^{5}$ Briggs, Lyman. Effect of Spin and Speed on Lateral Deflection (Curve) Of A Baseball; and the Magnus Effect for Smooth Spheres. 1st ed. Washington D.C: National Bureau of Standards, 1959. Web. 4 Feb. 2017.

${ }^{6}$ Patkunam, Kavithasan et al. EXPERIMENTAL STUDY OF MAGNUS EFFECT OVER AN AIRCRAFT WING.1st ed. Tamil Nadu: International Journal of Research in Engineering and Technology, 2017. Web. 4 Feb. 2017.
} 
The use of the coefficient of static friction is not completely accurate since even materials with a low surface roughness can have a higher coefficient due to the sticky nature of the material. For example, one material tested was rubber, which even though was very smooth to the touch the coefficient of static friction, came out to be 1.15. Another problem was the fact that this coefficient was found against a wood block, which acts differently to what air particles do. Proper apparatus could be used to measure this surface roughness to get better data.

\section{Random}

Even though there were attempts made to minimize the background air disturbances, obviously not all were stopped. The ideal setting would the use of a wind tunnel, which is a machine, used to emulate the flight motion in a confined space. However, it is very expensive and wasn't feasible to use.

\section{Systematic}

Since the mass of materials varied slightly, the inertial mass of the motor would be higher. This would result in a higher torque being needed to achieve the same angular velocity, resulting in slightly slower speeds for materials like 'felt'.

\section{Random}

As could be seen in Figure 4, the values at lower angular velocities did not fit any trend. This was, as explained above, due to the random errors, over-powering the actual data. However, as higher values are reached a clear pattern emerges since the random errors are now smaller in comparison. Taking readings with higher rotation speeds reduce the effect of the errors even further.

\section{Systematic}

The 'wobble' in the motor is due to the center of mass of the rotating mass not being perfectly centered. This could be avoided, by using better motors and more precise instruments, to cut and stick the cylinder.

\section{Systematic}

The weighing scale used was not designed for experiments like this. Due to this, the refresh rate (the rate at which mass readings are updated) was only around twice every second. This could result in an underestimation of the uncertainty values. The ideal device would be able to plot a graph of the mass readings too, so that uncertainty could be figured out in a much simpler way.

\section{REFERENCES}

1. "Boundary Layer". Grc.nasa.gov. Web. 7 Feb. 2017.

2. "Lift of a Rotating Cylinder". Grc.nasa.gov. N.p., 2017. Web. 4 Feb. 2017.

3. Richmond, Michael. "The Effect of Air on Baseball Pitches". Spiff.rit.edu. N.p., 2008. Web. 4 Feb. 2017.

4. "The Effect Of Air On Baseball Pitches". Thiiink.com. Web. 4 Feb. 2017.

5. "The Effect Of Surface Roughness On Flow Structures In A Neutrally Stratified Planetary Boundary Layer Flow". N.p., 2017. Web. 5 Feb. 2017.

6. "The Magnus Project". The Magnus Project. N.p., 2017. Web. 7 Feb. 2017.

7. "The Spinning Wing". Pilotfriend.com. N.p., 2017. Web. 4 Feb. 2017. 
8. "Heinrich Gustav Magnus Facts, Information, Pictures | Encyclopedia.Com Articles About Heinrich Gustav Magnus". Encyclopedia.com. N.p., 2017. Web. 7 Feb. 2017.

9. Briggs, Lyman. Effect of Spin and Speed On Lateral Deflection (Curve) Of A Baseball; And the Magnus Effect for Smooth Spheres. 1st ed. Washington D.C: National Bureau of Standards, 1959. Web. 4 Feb. 2017.

10. Bush, J.W.M. The Aerodynamics of the Beautiful Game. 1st ed. Web. 5 Feb. 2017.

11. Eric J, Sprigings. The Choice Between Bernoulli's or Newton's Model In Predicting Dynamic Lift. 1st ed. INTERNATIONAL JOURNAL OF SPORT BIOMECHANICS, 1990. Web. 5 Feb. 2017.

12. JoonAhn, Byung. Spin Rate and Deflection Ratio Of A Ping Pong Ball. 1st ed. ISB Journal of Science, 2013. Web. 7 Feb. 2017.

13. Marzuki, O. F. et al. AN EXPERIMENTAL INVESTIGATION ON THE EFFECT OF SURFACE ROUGHNESS ON THE PERFORMANCE OF MAGNUS WIND TURBINE. 1st ed. Asian Research Publishing Network, 2015. Web. 7 Feb. 2017.

14. Patkunam, Kavithasan et al. EXPERIMENTAL STUDY OF MAGNUS EFFECT OVER AN AIRCRAFT WING.1st ed. Tamil Nadu: International Journal of Research in Engineering and Technology, 2017. Web. 4 Feb. 2017.

15. Seifert, Jost. Progress In Aerospace Sciences. 1st ed. Manching: Elsevier, 2012. Web. 4 Feb. 2017.

16. Weiß, André and Anja Nicolai.THE UTILIZATION OF THE MAGNUS EFFECT FOR HOT WATER ROCKETS. 1st ed. Berlin: N.p. Web. 7 Feb. 2017.

17. Sarafian, Haiduke. Impact of The Drag Force And The Magnus Effect On The Trajectory Of A Baseball. 1st ed. Scientific Research Publishing Inc., 2015. Web. 4 Feb. 2017.

18. How To Curve A Ball Backwards Using Science Ft. FYFD. Physics Girl, 2016.video.

19. Seifert, Jost. Progress In Aerospace Sciences. 1st ed. Manching: Elsevier, 2012. Web. 4 Feb. 2017.

20. Surprising Applications Of The Magnus Effect. Veritasium, 2015.video.

21. The Physics Behind A Curveball - The Magnus Effect. Physics Girl, 2014.video.

22. Magnus Effect, Spinning Cylinder Wing (Rotor Wing) マグナス効果回転円筒翼.ken1 ken2kenken, 2013.video.

23. White, Frank M. Fluid Mechanics. 1st ed. New York: McGraw-Hill, 2008. Print.

24. Tsokos, K. A. Physics for The IB Diploma. 1st ed. Cambridge: Cambridge University Press, 2008. Print.

25. Audacity. Audacity, 2017. Print.

26. Air Density Calculator.Barani Design: N.p., 2012. Print. 
